\title{
RELATION INVOLVING $\psi$ FUNCTION AND I FUNCTION OF TWO VARIABLE
}

\author{
Sanjay Sharma ${ }^{1}$, Richa Gupta ${ }^{2}$, Bhupendra Tripathi ${ }^{3}$, Roshani Sharma ${ }^{4}$ \\ 1. Research Scholar, Department of School Education \\ Email: sanjaysharma3240@gmail.com \\ 2. Department of Mathematics. SRK University Bhopal \\ Email: richasharad.gupta@gmail.com \\ 3. Department of Mathematics, LNCT Bhopal \\ Email: tbhupendra79@gmail.com \\ 4. Department of Mathematics, LNCT Bhopal \\ Email: roshnipsharma@gmail.com
}

\begin{abstract}
In the paper, we established relation involving $\psi$ function and I- Function of two variable $I\left[z_{1}, z_{2}\right]$. Some more result known and new are also obtained as a particular cases of these relation.
\end{abstract}

Key Word: $\psi$ function. kampe de feriet function, I- function of two variable.

DOI: $10.7176 / \mathrm{MTM} / 9-4-08$

Publication date: April $30^{\text {th }} 2019$.

Recently number of relation involving $\psi$ function and I - Function in this paper, we use following method. Some relations involving the $\psi$ function and I- Function of two variable have been established. Further, more results involving kampe de feriet function and general hyper geometric series. We obtained as particular care of these relations.

\section{Definition and Notation:}

The multivariable extension of the kampe de fereit function in defined and represented as [ ].

$$
\begin{aligned}
& F_{i: m_{1} ; \ldots \ldots \ldots m_{n}}^{p: \ldots \ldots \ldots q_{n}}\left[z_{1}, \ldots \ldots \ldots, \mathrm{z}_{n}\right]=F_{i: m_{1} ; ; m_{n}}^{p: q_{1} ; q_{n}}\left[\begin{array}{l}
\left(a_{p}\right):\left(b_{q_{1}}^{(1)}\right) ; \ldots \ldots . . ;\left(b_{q_{n}}^{(n)}\right): \\
\left(\alpha_{l}\right):\left(\beta_{m_{i}}^{(l)}\right) ; \ldots \ldots . . ;\left(\beta_{m_{n}}^{(n)}\right)^{z_{1} \ldots \ldots . . \mathrm{z}_{n}}
\end{array}\right] . \\
& =\sum_{s_{1} \ldots s_{n}=0}^{\infty} g\left(s_{1}, \ldots \ldots \ldots, s_{n}\right) \frac{z_{1}^{s_{1}}}{s_{1} !} \ldots \ldots \frac{z_{n}^{s_{n}}}{s_{n} !} \\
& g\left(s_{1}, \ldots \ldots \ldots, s_{n}\right)=\frac{\prod_{j=1}^{p}\left(a_{j}\right)_{s_{1+\ldots}, s_{n}} \prod_{i=1}^{q_{1}}\left(b_{i}^{1}\right)_{s_{1}} \ldots \ldots \ldots \ldots . \prod_{s_{1}}^{q_{n}}\left(b_{i}^{(n)}\right)_{s_{n}}}{\prod_{i=1}^{l}\left(\alpha_{i}\right)_{s_{1+\ldots}+s_{n}} \prod_{i=1}^{m_{1}}\left(\beta_{i}^{1}\right) s_{1} \ldots \ldots \ldots \ldots . . \prod_{j=1}^{m_{n}}\left(\beta_{j}^{(n)}\right)_{s_{n}}}
\end{aligned}
$$

and for convergence $1+l+m_{k}-p-q_{k} \geq 0, k=1, \ldots . n$.

The equality hold when in addition, either $p>1$ and $\left|z_{1}\right|^{1 /(p-1)}+\ldots \ldots .+\left|z_{n}\right|^{1 /(p-1)}<1$ or $p \leq l$ and $\max \left\{\left|z_{1}\right|, \ldots \ldots \ldots .\left|z_{n}\right|\right\}<1$ 
The I- Function of two variable is defined by Sharma and Mishra [1991] represented by following manner:

$$
\begin{aligned}
& I\left[\begin{array}{c}
z_{1} \\
z_{2}
\end{array}\right]=I\left[z_{1}, z_{2}\right]=I_{p_{i}^{\prime} q_{i i r} ; p_{i}^{\prime}, q^{\prime}, r^{\prime} ; p_{i}^{\prime \prime}, q_{i}^{\prime \prime}, r^{\prime \prime}}\left[\begin{array}{l}
z_{1} \mid\left[\left(a_{j} ; \alpha_{j} ; A_{j}\right)_{1, n_{1}}\right],\left[\left(a_{j i} ; \alpha_{j i} ; A_{j i}\right)_{n+1, p_{i}}\right] ;\left[\left(e_{i}, E_{j}\right)_{1, n_{2}}\right]\left[\left(e_{j i}^{\prime \prime}, E_{j i}^{\prime \prime}\right)_{n_{1}+1, p_{2}^{\prime \prime}}\right] ;\left[\left(f_{j}, F_{j}\right)_{1, m_{1}}\right] \\
z_{2} \mid:\left[\left(c_{j}: y_{j}\right)_{1, n_{1}}\right]\left[\left(c_{j i} ; y_{j i}^{\prime}\right)_{n_{1}+1, p_{i}}\right] ;\left[\left(b_{j i} ; \beta_{j i} ; B_{j i}\right)_{1, q_{1}}\right]\left[\left(d_{j}, \delta_{j}\right)_{1, m_{1}}\right] ;\left[\left(d_{j i}, \delta_{j i}\right)_{m_{1}+1, q_{i}}\right]\left[\left(f_{j i}^{\prime \prime}, F_{j i}^{\prime \prime}\right)_{m_{2}+1, q_{i}}\right]
\end{array}\right. \\
& =\frac{1}{(2 \pi \omega)^{2}} \int_{L_{1}} \int_{L_{2}} \phi(\xi, \eta) \theta_{2}(\xi) \theta_{3}(\eta) z_{1}^{\xi_{1}} z_{2}^{\eta_{1}} d \xi d \eta \text {. where } \omega=\sqrt{-1} \text {. }
\end{aligned}
$$

$$
\phi(\xi, \eta)=\frac{\prod_{j=1}^{n}\left[\left(1-a_{j}+\alpha_{j} \xi+A_{j} \xi\right)\right]}{\sum_{i=1}^{n}\left[\prod_{j=n+1}^{p_{i}}\left[\left(a_{j i}-\alpha_{j i} \xi-A_{j i} \eta\right)\right] \prod_{j=1}^{q_{i}}\left[\left(1-f_{j i}^{\prime \prime}+F_{j i}^{n}\right)\right]\right]}=
$$

Along with other convergence condition as mention by Sharma and Mishra (1991). $z_{1}, z_{2}$ are not equal to zero and an empty product in interpreted as unity.

$$
\begin{aligned}
& \theta_{2}(\xi)=\frac{\prod_{j=1}^{n_{1}} \Gamma\left(1-c_{j}-\gamma_{j} \xi\right) \prod_{j=1}^{m} \Gamma\left(d_{j i}^{\prime}+\delta_{i} \xi\right)}{\sum_{i=1}^{n_{2}}\left[\prod_{j=n_{1}+1}^{p_{i}^{\prime}} \Gamma\left(c_{j i}-\gamma_{j i}\right) \prod_{j=m_{1+1}}^{\infty_{2}} \prod_{j=m_{1}+1}^{q_{i}^{\prime}} \Gamma\left(1-d_{j i}^{\prime}+\delta_{j i} \xi\right)\right]} . \\
& \theta_{3}(\eta)=\frac{\prod_{j=1}^{n_{2}} \Gamma\left(1-e_{j}-E_{j} \eta\right) \prod_{j=1}^{m_{2}} \Gamma\left(f_{j}-F_{j} \eta\right)}{\sum_{i^{\prime \prime}=1}^{r^{\prime \prime \prime}}\left[\prod_{j=n_{2}+1}^{p_{i}^{\prime \prime}} \Gamma\left(e_{j i}^{\prime \prime}-E_{j i}^{\prime \prime} \eta\right) \prod_{j=m_{2}+1}^{q_{i}^{\prime}} \Gamma\left(1-f_{j i}^{\prime \prime}+F_{j i}^{\prime \prime} \eta\right)\right]} .
\end{aligned}
$$

We required the following series representation of the I- function of two variable [4,pp-84-85] with the help of $\mathrm{H}$ - Function two variable on the simplify we get the definition of $\mathrm{H}$ - Function and the represented as summation series.

$I\left[z_{1}, \mathbf{z}_{2}\right]=\sum_{h=1}^{p_{2}} \sum_{h_{2}=1}^{p_{3}}\left\{\sum_{k=1}^{\infty} \sum_{k_{2}=0}^{\infty}(-1)^{k_{1}+k_{2}} \phi\left(\xi_{1}, \xi_{2}\right) \theta_{3}(\xi) \theta_{4}\left(\xi_{1}\right) x^{\xi_{1}} y^{\xi_{2}}\left(\left(\delta_{h_{1}}, f_{h_{2}}\right) k_{1} k_{2}\right)^{-1}\right\}$.

Where $\xi_{1}=\xi\left(h_{1}, k_{1}\right)=\frac{d_{n_{1}+k_{1}}}{\delta_{n_{1}}}$

and $\xi_{2}=\xi\left(h_{2}, k_{2}\right)=\frac{f_{n_{1}+k_{2}}}{F_{n_{2}}}$

If $\left\{{ }^{R} k_{1}, k_{2}\right\}_{k_{1}, k_{2=0}}^{\infty}$ in an arbitrary bounded sequence of complex number, we have

$$
\begin{aligned}
& =\sum_{n=1}^{\infty} \frac{(v)_{n}}{n(v)_{n}}\left\{\sum_{k_{1}=0}^{\infty} \sum_{k_{2}=0}^{\infty} \frac{R_{k_{1} k_{2}}}{(u+n)_{k_{1}+k_{2}}} \frac{z^{k_{1}+k_{2}}}{\left|k_{1}\right| k_{2}}\right\} \\
& =\sum_{k_{1}, k_{2}=0}^{\infty}\left[\frac{R_{k_{1} k_{2}}}{(u)_{k_{1}+k_{2}}}\left\{\psi\left(m+k_{1}+k_{2}\right)-\psi\left(u+k_{1}+k_{2}-v\right)\right\} \frac{z^{k_{1}+k_{2}}}{\left|k_{1}\right| k_{2}}\right]
\end{aligned}
$$

Again from [1] with above (1.10) and known Results

$$
\sum_{n=0}^{\infty} \frac{(a)_{n}(b)_{n}}{(c)_{n}}[\psi(c+n)-\psi(c)]=\frac{\Gamma(c) \Gamma(c-a-b)}{\Gamma(c-a) \Gamma(c-b)}[\psi(c-a)+\psi(c-b)-\psi(c)-\psi(c-a-b)]
$$


The following extension of the above given [1] to the case of two variable are obtained easily.

Let $\left\{R_{k_{1}}, R_{k_{2}}\right\}_{k_{1}, k_{2}=0}^{\infty}$ be an arbitrary sequence of complex number also let $u_{n}\{\mu, \rho ; z\}=\sum_{k_{1}=0}^{\infty} \sum_{k_{2}=0}^{\infty} \frac{\Gamma(\mu-\rho) \cdot z^{k_{1}+k_{2}}}{\Gamma(\mu+\eta+\rho) \underline{k_{1} \cdots . . k_{2}}}$.

Where $\rho=\rho\left(k_{1}, k_{2}\right)$ is a given with $\operatorname{Re}\left(\rho\left(k_{1}, k_{2}\right)\right) \geq 0$.

then $\sum \frac{(\alpha) n}{n} u_{n}(\mu, \rho ; z)=\sum_{k_{1}=0}^{\infty} \sum_{k_{2}=0}^{\infty}\left\{R_{k_{1}-k_{2}}[\psi(u+\rho)-\psi(-\alpha+u+\rho)] \frac{z^{k_{1}+k_{2}}}{\underline{k_{1}} \cdot \mid k_{2}}\right\}$.

Again let

$v_{n}(\alpha, \beta, \mu, \eta, \rho ; z)=\sum_{k_{1}=0}^{\infty} \sum_{k_{2}=0}^{\infty} R_{k_{1}, k_{2}} \frac{\Gamma(u+\rho) \Gamma(u+\alpha+\beta+\eta+\rho)}{\Gamma(u+\eta+\rho) \Gamma(u+\alpha+\beta+\rho+\eta+\rho)} \times 3 F_{2}\left[\begin{array}{c}\alpha+\eta, \alpha+\rho ;-n \\ \mu+\alpha+\beta+\eta+\rho ; \alpha\end{array} ; 1\right] \frac{z^{k_{1}+k_{2}}}{\left|k_{1}\right| k_{2}}$.

\section{Main Results:}

We establish the following results:

$$
\begin{aligned}
& \sum_{n=1}^{\infty} \frac{(\alpha) n}{n} I_{p_{i}, q_{i}: r ; p_{i}, q_{i} ; p_{i}, q_{i}, r^{\prime}}^{o, n}\left[\begin{array}{c|c}
z_{1} \mid \\
z_{2}
\end{array} \mid \begin{array}{c}
\left(q_{i}, u_{j}, c_{1}, s_{2}\right),\left(a_{j}, \alpha_{i}, A_{j}\right) \\
\left(b_{i}, \beta_{j}, B_{j}\right)_{1, s_{1}}\left(1-u-n ; c_{1}, c_{2}\right)
\end{array}\right] . \\
& =\sum_{h_{1}=1}^{b_{2}} \sum_{h_{3}=1}^{b_{3}}\left\{\sum_{k_{1}=0}^{\infty} \sum_{k_{2}=0}^{\infty}(-1)^{k_{1}+k_{2}} \phi\left(\xi_{1} \xi_{2}\right) \theta_{3}\left(\xi_{1}\right) \theta_{4}\left(\xi_{2}\right)\left(s_{n_{1}}, F_{h_{2}}\right)^{-1} \times\left[\psi\left(u+a \xi_{1}+c_{2} \xi_{2}\right)-\psi\left(-\alpha+u+c_{1} \xi_{1}+c_{2} \xi_{2}\right)\right] \frac{z^{\xi_{1}+\xi_{2}}}{k_{1}}\right\}
\end{aligned}
$$

\section{Proofs of Main Result}

To Establish (2.1), We assume

$$
p\left(k_{1}, k_{2}\right)=c_{1} \xi_{1}+c_{2} \xi_{2} \quad\left(c_{1}, c_{2} \text { are constants }\right)
$$

and $\xi_{1}, \xi_{2}$ are given by (1.7 and (1.8)

Next we assume

$$
R_{k_{1}, k_{2}}=(-1)^{k_{1}+k_{2}} \phi\left(\xi_{1}, \xi_{2}\right) \theta_{3}\left(\xi_{1}\right) \theta_{4}\left(\xi_{2}\right)\left(\delta_{h_{1}} F_{h_{2}}\right)^{-1} .
$$

Where $\phi\left(\xi_{1}, \xi_{2}\right), \theta_{3}\left(\xi_{1}\right)$ and $\theta_{4}\left(\xi_{2}\right)$ are given by (1.5) and (1.9), respectively. Now we multiply both sides of (1.9) by $z^{\xi_{1}+\xi_{2}-k_{1}-k_{2}}$ and then sum up the resulting equation from $h_{1}=1$ to $p_{2}$ and $h_{2}=1$ to $p_{3}$ and then interpret with the help of (1.6) to arrive at the result given in $(2.1)$ 
To establish (2.2), we again assume that $p=p\left(k_{1}, k_{2}\right)=c_{1} \xi_{1}+c_{2} \xi_{2}, \quad\left(c_{1}, c_{2}\right.$ are constants) and $\xi_{1}, \xi_{2}$ are given by (1.7) and (1.8). In new of the series representation of $H\left[\begin{array}{l}x \\ y\end{array}\right]$, given in (1.6), we further let

$$
R_{k_{1}, k_{2}}=(-1)^{k_{1}+k_{2}} \phi\left(\xi_{1}, \xi_{2}\right) \theta_{3}\left(\xi_{1}\right) \theta_{4}\left(\xi_{2}\right)\left(\delta_{h_{1}}, F_{h_{2}}\right)^{-1}
$$

Where $\phi\left(\xi_{1}, \xi_{2}\right)$ and $\theta_{3}\left(\xi_{1}\right)$ are given by (1.5) and (1.9), in (1.21) and then multiply both sides of (1.21) by $z^{\xi_{1}+\xi_{2}-k_{1}-k_{2}}$ and sum up both sides of the resulting equation from $h_{1}=1$ to $p_{2}$ and $h_{2}=1$ to $p_{3}$ and then interpret with the help of (1.6) to arrive at result (2.2)

Similarly, the result in (2.3) is established with the help of (1.22) and (1.23)

\section{Reference}

1. R. G. Bushehman and K. C. Gupta: Contigeses relation for the H - Function of the variables, Indian I pure. Appl. Math b (1973), 1416 - 1421.

2. K. G. Gupta and O. P. Garg: on finite summation formulae for the $\mathrm{H}$ - function of two variable, kyungpook math. J. 18 (1978), $211-215$.

3. K. G. Gupta and O. P. Garg: on certain finite series involving the $\mathrm{H}-$ function of two variable Rev. Tech. Fac. Ingn. Units zutia 2(1979) $56-81$.

4. H. M. Shrivastava and M. C. Daaust: certain generalize new mann expansion associative with kampe de' ferret function, neden. Akad. Wetansh, pure, sor. A92 - mdag. Math 31(9169) (449-459)

5. H. M. Shrivastava, K. C. Gupta and S. P. Goyal: The H - function of one and two variable with applications, South Asian Publishers, New Delhi and Madras (1982).

6. H. M. Shrivastava and R. Panda: Some Bilateral generation function for a class of generalized hyper geometric polynomials. Raine Angew. Math 283/284(1976), 265-270.

7. Sharma, C. K and Panday (N. K) (1993): Certain expansion formulae, bull of pure and applied science. 12E(1-2); P 37-40.

8. Sharma, C. K. and Mishra, G - K (1994): Some identities involving the multivariable I - function bull of pure and applied science vol. B (E), No. 1, P $63-68$.

9. Chen, K. Y. and Srivastava, H.M., Some infinite series and functional relations that arose in the context of fractional calculus, J. Math, Anal, Appl., 252, (2000), 376-388.

10. Kalla, S.L. and Ross, B., Fractional Calculus, Pitman Advanced Publishing Program, 1985.

11. Kant, S. and Koul, C.L. On functional relations, Proc. Nat. Acad. Sci. India, 61 (A) II, 1991.

12. Srivastava, H.M., Gupta, K.C. and Goyal S.P., The H - Functions of One and Two Variables, South Asian Publishers, New Delhi, 1982.

13. Srivastava, H.M. and Manocha, H.L., A Treatise on Generating Functions, John Wiley and Sons, New York, 1984. 